\title{
National asset map for clinical trials launched
}

$\mathrm{C}$ anada is aiming to regain its research edge through a new national database highlighting the country's resources for clinical trials in the hope it will attract international companies doing research.

The Canadian Clinical Trials Asset Map (CCTAM) website went live June 4 with over 800 database records, and it is expected to grow. The map provides information on conditions and diseases being studied, and identifies gaps. For example, a search for cardiology in the system, would allow you to see all the clinical sites involved in research in that domain on a Google map. The map also documents an investigator's specialty, trial phase and the patient population. Registration is free, although user accounts have to be pre-approved by an administrator.

The designers of the asset map say that there is oversight to ensure the information about the research sites is accurate and up to date. The website's software finds older records and asks the owner of of the records to provide more recent data, Dr. Shurjeel Choudhri, senior vice president and head, Medical and Scientific Affairs, Bayer Inc., told CMAJ.

"Our goal is to have something that is sustainable. It has mechanisms built within it to maintain and keep itself updated so it will be a living document that will be available to Canadians for a long time to come," said Choudhri.

The asset map was funded and led by multiple partners, including the Canadian Institutes of Health Research, Rx\&D and the advocacy organization, HealthCareCAN.

Conservative Senator, Kelvin Ogilvie, a renowned biotechnologist and researcher observed that "the number of Canadian clinical trials has been declining for over a decade."

Ogilvie hopes the map will "at the very least stem the decline." However, Canada faces many barriers to becoming a research leader. Not the least of these is the unwillingness of research centres to set common requirements for clinical trials. This inhibits our ability to compete with other countries in a timely fashion.

The asset map is the first tangible outcome of the 2011 Canadian Clinical

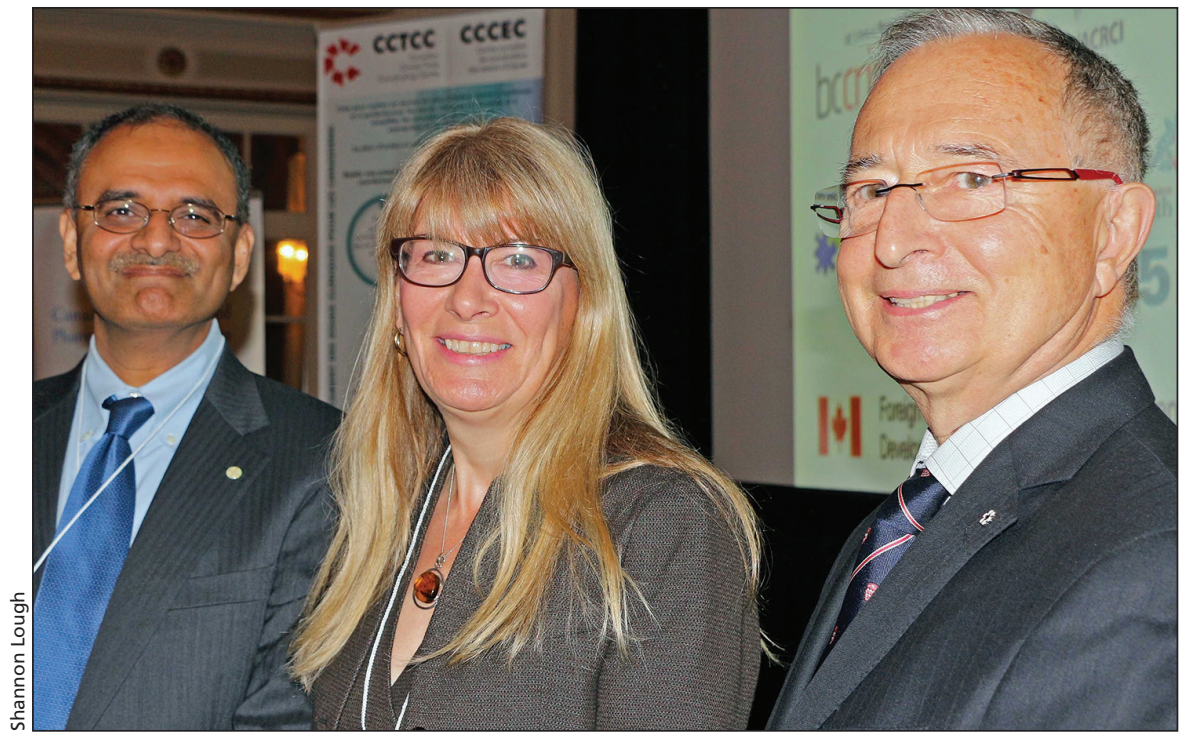

The Canadian Clinical Trials Asset Map was launched by researchers, industry leaders and policy-makers including, left to right, Dr. Shurjeel Choudhri, Bayer Inc., Belinda Vandersluis, director of the Canadian Clinical Trials Coordinating Centre, and Senator Kelvin Ogilvie.

Trial Summit where research-industry leaders developed a plan for improving the ability to conduct research in Canada. The summit concluded that Canada's system is expensive and that having numerous ethical boards hinders the timeliness of trial start-ups. The online database may not speed up ethical review boards for trials, but at least all the different components involved in running a clinical trial in Canada will be available in one place.

Belinda Vandersluis, the director of the Canadian Clinical Trials Coordinating Centre (CCTCC) said Canada has a long-time reputation for conducting practice-changing, high-quality clinical trials, with internationally renowned researchers. "We don't want to lose the reputation, the researchers, or the opportunity to study innovative and important new drugs, devices and vaccines."

The CCTCC was established in April 2014 with a mandate to improve the clinical-trial landscape. Its $\$ 1.5$ million in funding came from the same partners as the asset map. Although it only has a three-year directive, the national database will continue to run.

Pharmaceutical companies were active partners in designing the national asset map. Choudhri offered advice on how Canada can compete with other countries. "We typically have only a few weeks to identify potential sites for the trial, determine the patient population needed for that trial. By having this tool you can do a quick search and identify the sites that are active in that particular area."

Before the national map was created, there were about 25 smaller asset maps in Canada, said Choudhri, but the data was fragmented among federal, provincial and regional bodies and some of the information was only available on paper.

"All had some gaps and a lot of them were out of date. Those were the inspiration for thinking we needed something better at a national level."

The most up-to-date online maps were in Ontario and British Columbia. The BC Clinical Research Infrastructure Network partnered with the asset map project in offering its data. Director of Operations Heather Harris said the collaboration among provinces highlights Canada's strengths as a whole rather than having separate agendas. "I think that it's important to speak with that national voice."

The asset map isn't only for global marketing, said CCTAM Project Manager Elena Aminkova; it's also for policy-makers. Users will soon be able to search by federal electoral boundaries as well. — Shannon Lough, CMAJ

CMAJ 2015. DOI:10.1503/cmaj.109-5089 